\title{
MULTIPLICATION OF NATURAL NUMBER PARAMETERS AND EQUATIONS IN A FREE SEMIGROUP
}

\author{
GENNADY S. MAKANIN
}

\begin{abstract}
This paper deals with the problem of describing the set $M$ of all solutions of an equation over a free semigroup $S$. The standard way to do this involves the introduction of auxiliary equations containing polynomials in natural number parameters of arbitrarily high degree. Since $S$ has a solvable word problem, $M$ must be computable. However, $M$ cannot necessarily be computed from the standard description of $M$. The present paper shows that the only polynomials needed to describe $M$ are just products of one parameter by a linear combination of some other parameters. The resulting simplification of the standard description of $M$ clearly can be used to compute $M$.
\end{abstract}

Investigating the general solution of a one variable equation in a free group, $\mathrm{R}$. Lyndon [1], [2] was the first who faced the necessity of introducing the natural number parameters for the auxiliary equations whose solutions are being used for the construction of the solutions of the initial equation. The difficulty arising in such investigations is the following: the multiplication of the natural number parameters leads to nonlinear Diophantine equations and the decision problem for these equations is algorithmically unsolvable as Yu. V. Matiyasevich has proved in [3]. In the papers of R. Lyndon [1], [2], Yu. I. Khmelevskii [4], [5] and others, where equations with few variables were being studied, multiplication of the natural number parameters had been avoided by imposition of additional conditions on the parameters and the bases. However, in the general case, the investigation of equations with an arbitrary number of variables in a free group or a free semigroup inevitably implies the introduction of natural number parameters and multiplication of the natural number parameters introduced.

In this paper we propose radical deliverance from the aforesaid difficulty. We prove that the product of the parameters in the auxiliary equations of the tree of the transformations occurs in the form of a product $L \lambda$ where $L$ is a linear polynomial of natural number parameters which are different from $\lambda$, and the parameter $\lambda$ enters into the equation only in the form $L \lambda$. The transformation of the normalization of the parameters of the equation is introduced. It consists of the replacement of the product $L \lambda$ by the factor $\lambda$. We note this transformation increases the set of the solutions of the equation in general.

The transformation of the normalization of the parameters allows us to formulate in a way which is free from multiplication of the parameters the concept of the parametric equation in a free semigroup, which came to a head long ago.

Received by the editors November 2, 1994.

1991 Mathematics Subject Classification. Primary 20M05; Secondary 03D40, 20F10.

Supported by the American Mathematical Society and Russian Foundation for Fundamental Research. 
Using the transformation of the normalization of the parameters we construct a new tree of auxiliary parametric equations in a free semigroup and prove that the set of the solutions of the initial equation in a free semigroup does not change thereby.

Let $\Pi$ be a free semigroup with a countable alphabet of generators

$$
a_{1}, \ldots, a_{k}, \ldots \text {. }
$$

An equation in $\Pi$ is given by an alphabet of word variables

$$
x_{1}, \ldots, x_{n}
$$

and a left noncancellable equality

$$
\varphi\left(x_{1}, \ldots, x_{n}\right)=\psi\left(x_{1}, \ldots, x_{n}\right) .
$$

A list $X_{1}, \ldots, X_{n}$ of words in the alphabet (1) is called a solution of the equation (2), (3) whenever the words $\varphi\left(X_{1}, \ldots, X_{n}\right)$ and $\psi\left(X_{1}, \ldots, X_{n}\right)$ coincide. If $\varphi\left(x_{1}, \ldots, x_{n}\right)$ and $\psi\left(x_{1}, \ldots, x_{n}\right)$ are empty words, the equation $(2),(3)$ is called trivial. Coincidence of two words $P$ and $Q$ will be denoted by $P=Q$.

Two types of elementary transformations of the equation (2), (3) called nondegenerate and degenerate elementary transformations, are defined: The nondegenerate transformation $x_{p} \rightarrow x_{q} x_{p}$, where $p \neq q$, can be applied to the equation (2), $x_{p} \varphi_{1}\left(x_{1}, \ldots, x_{n}\right)=x_{q} \psi\left(x_{1}, \ldots, x_{n}\right)$. The result of the application of this transformation is given by the alphabet (2) and the equality $x_{p}\left(\varphi_{1}\right)^{x_{p} \rightarrow x_{q} x_{p}}=$ $\left(\psi_{1}\right)^{x_{p} \rightarrow x_{q} x_{p}}$. The degenerate transformation $x_{p} \rightarrow 1$ can be applied to the equation (2), $x_{p} \varphi_{1}\left(x_{1}, \ldots, x_{n}\right)=\psi\left(x_{1}, \ldots, x_{n}\right)$. The result of the application of this transformation is given by the alphabet $x_{1}, \ldots, x_{p-1}, x_{p+1}, \ldots, x_{n}$ and the equality $\left(\varphi_{1}\right)^{x_{p} \rightarrow 1}=(\psi)^{x_{p} \rightarrow 1}$ after all possible left cancellations.

A sequence of equations

$$
E \rightarrow E_{1} \rightarrow \cdots \rightarrow E_{\tau}
$$

connected by elementary transformations is called a finished sequence of the equation $E$ if the equation $E_{\tau}$ is trivial.

The transformation

$$
\left\{\begin{array}{c}
x_{1} \rightarrow W_{1}\left(x_{1}, \ldots, x_{n}\right) \\
\ldots \ldots \ldots . \ldots . . . . . \\
x_{n} \rightarrow W_{n}\left(x_{1}, \ldots, x_{n}\right)
\end{array}\right.
$$

resulting from all successive applications of elementary transformations in any finished sequence of the equation (2), (3) is called a principal solution of the equation (2), (3).

The following theorem demonstrates that the general solution of any equation in $\Pi$ is described by the set of all principal solutions of this equation.

Theorem 1. For each solution $X_{1}, \ldots, X_{n}$ of the equation (2), (3) there exists a principal solution (5) and a list $L_{1}, \ldots, L_{n}$ of words in the alphabet (1) such that $X_{i}=W_{i}\left(L_{1}, \ldots, L_{n}\right)$ for all $i=1, \ldots, n$. On the other hand for each principal solution (5) the words $\varphi\left(W_{1}\left(x_{1}, \ldots, x_{n}\right), \ldots, W_{n}\left(x_{1}, \ldots, x_{n}\right)\right)$ and $\psi\left(W_{1}\left(x_{1}, \ldots, x_{n}\right), \ldots, W_{n}\left(x_{1}, \ldots, x_{n}\right)\right)$ coincide.

Proof. Let the equality (3) be of the form $x_{1} \varphi_{1}\left(x_{1}, \ldots, x_{n}\right)=x_{2} \psi_{1}\left(x_{1}, \ldots, x_{n}\right)$. If $X_{i}=1(i=1,2)$, then the solution $X_{1}, \ldots, X_{n}$ induces the transformation $x_{i} \rightarrow 1$. If $\partial\left(X_{1}\right) \geq \partial\left(X_{2}\right)>0$, then $X_{1}=X_{2} X_{1}^{\prime}$ for some word $X_{1}^{\prime}$ and the solution induces 
the transformation $x_{1} \rightarrow x_{2} x_{1}$. If $\partial\left(X_{2}\right)>\partial\left(X_{1}\right)>0$, then the solution induces the transformation $x_{2} \rightarrow x_{1} x_{2}$. Let the equality (3) be of the form $x_{1} \varphi\left(x_{1}, \ldots, x_{n}\right)=1$. Then $X_{1}=1$ and the solution induces the transformation $x_{1} \rightarrow 1$. Applying this transformation, we obtain a new equation and solution of this equation constructed from $X_{1}, \ldots, X_{n}$ by means of suitable transformation. Repeat this operation. Since each application of the operation diminishes either the number of components in a solution or the length of a component of the solution, after a finite number of steps we arrive at the trivial equation.

The equality $\varphi\left(W_{1}, \ldots, W_{n}\right)=\psi\left(W_{1}, \ldots, W_{n}\right)$ follows from definition of principal solution.

Starting from the initial equation (2), (3) we "construct" the infinite tree of auxiliary equations by successive application of all possible elementary transformations.

A sequence of nondegenerate transformations $x_{k} \rightarrow x_{i_{1}} x_{k}, x_{k} \rightarrow x_{i_{2}} x_{k}, \ldots, x_{k} \rightarrow$ $x_{i_{m}} x_{k}$ applied to an equation of the form $x_{k} \varphi\left(x_{1}, \ldots, x_{n}\right)=x_{i_{1}} x_{i_{2}} \ldots$ $x_{i_{m}} x_{k} \psi\left(x_{1}, \ldots, x_{n}\right)$ is called a stable sequence of transformations of one variable.

If in the tree of auxiliary equations of the initial equation (2), (3) there is a stable sequence of transformations of one variable, it is obvious that there is a sequence of nondegenerate transformations of any length in it. To exclude stable sequences from the tree, we are forced to collect them in special parametric transformations of the form

$$
x_{k} \rightarrow\left(x_{i_{1}} x_{i_{2}} \cdots x_{i_{m}}\right)^{\lambda} x_{k}
$$

where $\lambda$ is a natural parameter. Thus, we extend the notion of equation in the free semigroup, also extend the notion of transformation of such equation and as a result we come to the notion of parametric equation in the free semigroup.

We introduce the countable table of the natural parameters.

$$
\begin{aligned}
& \lambda_{1,1}, \ldots, \lambda_{1, w}, \ldots \\
& \ldots \ldots \ldots \ldots \ldots \\
& \lambda_{p, 1}, \ldots, \lambda_{p, w}, \ldots \\
& \ldots \ldots \ldots . \ldots .
\end{aligned}
$$

The set of all linear polynomials of the form $k_{0}+\sum_{i=1}^{r} k_{i} \lambda_{p, i}$, where $r, k_{0}, k_{1}, \ldots, k_{r}$ are natural numbers, and $\lambda_{p, 1}, \ldots, \lambda_{p, r}$ are natural parameters from the $p$ th row of the table $(6)$, will be denoted by $\mathfrak{L}_{p}$.

The transformation of natural parameters $\lambda_{s, t} \rightarrow L_{s, t}(1 \leq s \leq p ; 1 \leq t \leq w)$, where $L_{s, t} \in \mathfrak{L}_{s}$ will be called row transformation.

A word $C$ on the alphabet (2) is called a simple word, if there exists no word $D$ such that $C=D^{m}$ with $m>1$. In particular, a simple word is not empty.

Consider the table of parametric words

$$
\begin{aligned}
& x_{1}, \ldots, x_{n} \\
& C_{1}^{\lambda_{1,1}}, \ldots, C_{1}^{\lambda_{1, w}}, \ldots \\
& \ldots \ldots \ldots \ldots \ldots \ldots \ldots \ldots \ldots \ldots \\
& C_{p}^{\lambda_{p, 1}}, \ldots, C_{p}^{\lambda_{p, w}}, \ldots,(p \geq 0)
\end{aligned}
$$


where $C_{1}$ is a word on the alphabet $x_{1}, \ldots, x_{n}$ and where for each $q=1, \ldots, p-1$ $C_{q+1}$ is a word in the alphabet

$$
\begin{aligned}
& x_{1}, \ldots, x_{n} \\
& C_{1}^{\lambda_{1,1}}, \ldots, C_{1}^{\lambda_{1, w}}, \ldots \\
& \ldots \ldots \ldots . \cdots \\
& C_{q}^{\lambda_{q, 1}}, \ldots, C_{1}^{\lambda_{q, w}}, \ldots
\end{aligned}
$$

Every word of the table (7) is called a parametric letter. The alphabet (7) is called the $p$-layer alphabet of parametric letters. The word $C_{i}(i=1, \ldots, p)$ is called the base of the letters of the $i$ th layer of the alphabet (7). We assume that two words in the alphabet (7) coincide if one obtains from another by commutations of parametric words the same layer and commutations parametric letter and its base. Coincidence of two words $P$ and $Q$ will be denoted $P=Q$. A parametric letter $C_{r}^{\lambda_{r, i}}$ is called a normal parametric letter, if for any value of parameters the base $C_{r}$ remains a simple word.

A parametric equation in a free semigroup $\Pi$ is given by the $p$-layer alphabet of the parametric letters (7) and a left noncancellable equality of words

$$
\Phi\left(x_{1}, \ldots, x_{n}, \ldots \lambda_{s, t} \ldots\right)=\Psi\left(x_{1}, \ldots, x_{n}, \ldots \lambda_{s, t} \ldots\right)
$$

in this alphabet. A list of words $X_{1}, \ldots, X_{n}$ in the alphabet (1) and a finite table of natural numbers $\left\{\cdots \Lambda_{s, t} \cdots\right\}$ is called a solution of the parametric equation (7), (8) whenever the words $\Phi\left(X_{1}, \ldots, X_{n}, \ldots \Lambda_{s, t} \ldots\right)$ and $\Psi\left(X_{1}, \ldots, X_{n}, \ldots \Lambda_{s, t} \ldots\right)$ coincide.

A parametric equation, given by the alphabet of normal parametric letters and an equality of words of the form

$$
x_{r} \Phi\left(x_{1}, \ldots, x_{n}, \ldots \lambda_{s, t} \ldots\right)=\Psi\left(x_{1}, \ldots, x_{n}, \ldots \lambda_{s, t} \ldots\right)
$$

in this alphabet, is called a normal parametric equation.

A list of row transformations of the natural parameters of the $p$ th row $\lambda_{p, 1}, \ldots$, $\lambda_{p, w}$

$$
\left\{\begin{array}{c}
\lambda_{p, 1} \rightarrow L_{1,1} \\
\ldots \ldots \ldots \ldots \\
\lambda_{p, w} \rightarrow L_{1, w}
\end{array} \quad, \ldots,\left\{\begin{array}{c}
\lambda_{p, 1} \rightarrow L_{\sigma, 1} \\
\ldots \ldots \ldots \ldots \\
\lambda_{p, w} \rightarrow L_{\sigma, w}
\end{array}\right.\right.
$$

will be called:

-full; if for each vector of natural numbers $l_{1}, \ldots, l_{w}$ there exists $t$ such that the system

$$
\left\{\begin{array}{c}
L_{t, 1}=l_{1} \\
\ldots \ldots \ldots \\
L_{t, w}=l_{w}
\end{array}\right.
$$

has a solution;

-independent, if for any $r, s(r \neq s)$ the system

$$
\left\{\begin{array}{c}
L_{r, 1}=L_{s, 1} \\
\ldots \ldots \ldots \ldots \\
L_{r, w}=L_{s, w}
\end{array}\right.
$$

has no solution. 
A list of row transformations of the natural parameters of the finite table

$$
\begin{aligned}
& \lambda_{1,1}, \ldots, \lambda_{1, w} \\
& \ldots \ldots . . \\
& \lambda_{t, 1}, \ldots, \lambda_{t, w}
\end{aligned}
$$

will be called a full (independent) list, if in the list of transformations every row of the table is full (independent).

Three types of transformations of the natural parameters of the parametric equation are defined.

A transformation of the parametric equation (7), (8), that we call a division of the natural parameters, is produced by means of a full and independent list of row transformations $\omega_{1}, \ldots, \omega_{\sigma}$ of natural parameters. The result of the application of transformation is a list of parametric equations $(7)^{\omega_{1}},(8)^{\omega_{1}} ; \cdots ;(7)^{\omega_{\sigma}}$, $(8)^{\omega_{\sigma}}$. The alphabet $(7)^{\omega_{i}}$ is obtained from the alphabet (7) by substituting the bases $C_{1}, \ldots, C_{p}$ by the words $C_{1}^{\omega_{i}}, \ldots, C_{p}^{\omega_{i}}$ respectively. The equality (8) ${ }^{\omega_{i}}$ is obtained from the equality $\Phi^{\omega_{i}}=\Psi^{\omega_{i}}$ by left cancellations.

A transformation of parametric equation (7), (8), that we call a replacement of the natural parameters, is applied to the equation when $C_{q}=C_{s}(1 \leq s<q \leq p)$ on the alphabet (7). This transformation is produced by means of transformation of the natural parameters

$$
\left\{\begin{array}{c}
\lambda_{q, 1} \rightarrow \lambda_{s, w+1} \\
\ldots \ldots \ldots \ldots \\
\lambda_{q, w} \rightarrow \lambda_{s, w+w}
\end{array}\right.
$$

where $w$ is maximal second index of natural parameters $\lambda_{i, j}$ that occurs in the words $\Phi, \Psi$, and bases $C_{1}, \ldots, C_{p}$. In addition the replacement of every letter $C_{q}^{\lambda_{q, k}}$ by the letters $C_{s}^{\lambda_{s, k+w}}$ respectively is carried out.

A transformation of parametric equation (7), (8) that we call a normalization of the natural parameter, is applied to the equation when the bases $C_{1}, \ldots, C_{m-1}$ are normal; when $C_{m}=C_{s}^{L_{s}+1}$ where $s<m, L_{s} \in \mathfrak{L}_{s}$; when the bases $C_{m+1}, \ldots, C_{p}$ and words $\Phi, \Psi$ contain a single letter of the $m$ th layer $C_{m}^{\lambda_{m, 1}}$. This transformation is produced by means of transformation of the natural parameters

$$
\left(L_{s}+1\right) \cdot \lambda_{m, 1} \rightarrow \lambda_{m, 1} .
$$

In addition in the equation (7), (8) the replacement the base $C_{m}$ by the base $C_{s}$ is carried out.

Three types of the transformation of the word variables of the parametric equation are defined.

A nondegenerate transformation is applied to normal parametric equation, given by the alphabet (7) and equality of the form

$$
x_{r} \Phi\left(x_{1}, \ldots, x_{n}, \ldots \lambda_{s, t} \ldots\right)=R \Psi\left(x_{1}, \ldots, x_{n}, \ldots \lambda_{s, t} \ldots\right),
$$

where $R$ is a parametric letter not containing $x_{r}$. A nondegenerate transformation is of the form $x_{r} \rightarrow R x_{r}$. The result of the application of the transformation is the parametric equation, given by the alphabet $(7)^{x_{r} \rightarrow R x_{r}}$ and the equality

$$
x_{r}(\Phi)^{x_{r} \rightarrow R x_{r}}=(\Psi)^{x_{r} \rightarrow R x_{r}} .
$$


A degenerate transformation is applied to a normal parametric equation, given by the alphabet (7) and equality of the form

$$
x_{r} \Phi\left(x_{1}, \ldots, x_{n}, \ldots \lambda_{s, t} \ldots\right)=\Psi\left(x_{1}, \ldots, x_{n}, \ldots \lambda_{s, t} \ldots\right) .
$$

A degenerate transformation is of the form $x_{r} \rightarrow 1$. The result of the application of the transformation is a parametric equation, given by the alphabet $(7)^{x_{r} \rightarrow 1}$ and the equality $(\Phi)^{x_{r} \rightarrow 1}=(\Psi)^{x_{r} \rightarrow 1}$ after left cancellations.

A parametric transformation is applied to a normal parametric equation, given by the alphabet (7) and equality of the form

$$
x_{r} \Phi\left(x_{1}, \ldots, x_{n}, \ldots \lambda_{s, t} \ldots\right)=Q x_{r} \Psi\left(x_{1}, \ldots, x_{n}, \ldots \lambda_{s, t} \ldots\right),
$$

where $Q$ is nonempty parametric word not containing $x_{r}$. A parametric transformation is of the form $x_{r} \rightarrow Q^{\lambda_{p+1,1}} x_{r}$. The result of the application of this transformation is a parametric equation, given by the alphabet that is obtained from the alphabet (7) by substituting for the bases $C_{1}, \ldots, C_{p}$ the words $C_{1}^{x_{r} \rightarrow Q^{\lambda_{p+1,1} x_{r}}}, \ldots$, $C_{p}^{x_{r} \rightarrow Q^{\lambda_{p+1,1 x_{r}}}}$ respectively, by addition of a row of letters

$$
Q^{\lambda_{p+1,1}}, \ldots, Q^{\lambda_{p+1, w}}, \ldots
$$

and the equality

$$
x_{r}(\Phi)^{x_{r} \rightarrow Q^{\lambda_{p+1,1} x_{r}}}=Q x_{r}(\Psi)^{x_{r} \rightarrow Q^{\lambda_{p+1,1} x_{r}}} .
$$

Remark. The definition of a parametric equation in a free semigroup given above is rather complicated, due to the intrinsic requirements of the definition. In practice the following simple definition should be used, which is equivalent to the aforesaid one:

Let $\lambda_{1}, \ldots, \lambda_{k}, \ldots$ be a countable alphabet of natural parameters. Define inductively a parametric word as follows: Any word on the alphabet (2) is a parametric word. If $P$ is a parametric word, then $(p)^{\lambda_{k}}$ is a parametric word. If $P$ and $Q$ are parametric words, then $P Q$ is a parametric word.

Every letter of the alphabet (2) and every parametric word of the form $(p)^{\lambda_{k}}$ is called a parametric letter. A parametric word is called a right parametric word, if for any letters $(C)^{\lambda_{q}}$ and $(D)^{\lambda_{q}}$, contained in this word, the parametric words $C$ and $D$ coincide. A parametric equation in a free semigroup is given by an alphabet (2) and equality of right parametric words.

A sequence of nondegenerate transformations

$$
x_{k} \rightarrow R_{1} x_{k}, x_{k} \rightarrow R_{2} x_{k}, \ldots, x_{k} \rightarrow R_{m} x_{k},
$$

applied to a parametric equation of the form

$$
x_{k} \Phi\left(x_{1}, \ldots, x_{n}, \ldots \lambda_{s, t} \ldots\right)=R_{1} R_{2} \ldots R_{m} x_{k} \Psi\left(x_{1}, \ldots, x_{n}, \ldots \lambda_{s, t} \ldots\right)
$$

where $R_{1}, R_{2}, \ldots, R_{m}$ are parametric words, is called a stable sequence of transformations of one variable.

Consider the sequence of parametric equations

$$
E_{0} \rightarrow E_{1} \rightarrow \cdots \rightarrow E_{i-1} \rightarrow E_{i} \rightarrow \cdots \rightarrow E_{\tau},
$$

where $E_{0}$ is the initial equation (2), (3) and for each $i=1,2, \ldots, \tau$ the equation $E_{i}$ is given by the $p$-layer alphabet of parametric letters and the equality

$$
\Phi_{i}\left(x_{1}, \ldots, x_{n}, \ldots \lambda_{s, t} \ldots\right)=\Psi_{i}\left(x_{1}, \ldots, x_{n}, \ldots \lambda_{s, t} \ldots\right) .
$$


Suppose for each $i=1,2, \ldots, \tau$ the couple $E_{i-1} \rightarrow E_{i}$ is connected by transformation $\omega_{i}$. Suppose the sequence of the parametric equations (9) does not contain any stable sequences of transformations of one variable.

The sequence of parametric equations (9) identically defines the principal transformation $\Omega_{i}(i=1,2, \ldots, \tau)$ of the initial equation (2), (3)

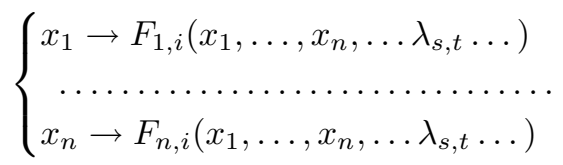

where $F_{1,0}=x_{1}, \ldots, F_{n, 0}=x_{n} ;$ and for each $i=1,2, \ldots, \tau$ the words $F_{1, i}, \ldots, F_{n, i}$ are obtained from the words $F_{1, i-1}, \ldots, F_{n, i-1}$ respectively by means of the transformation $\omega_{i}$.

The principal transformation $\Omega_{\tau}$ is called a principal parametric solution of the initial equation (2), (3) if $E_{\tau}$ is the trivial equation.

Theorem 2. For every solution $X_{1}, \ldots, X_{n}$ of the initial equation (2), (3) there exists a principal parametric solution $\Omega_{\tau}$, a list $K_{1}, \ldots, K_{n}$ of words in the alphabet (1) and a finite table of natural numbers $\left\{\cdots \Lambda_{s, t} \cdots\right\}$ such that $X_{r}=$ $F_{r, \tau}\left(K_{1}, \ldots, K_{n}, \ldots, \Lambda_{s, t} \ldots\right)$ for all $r=1, \ldots, n$. On the other hand for each principal parametric solution $\Omega_{\tau}$ of the equation (2), (3) the words $\varphi\left(F_{1, \tau}, \ldots, F_{n, \tau}\right)$ and $\Psi\left(F_{1, \tau}, \ldots, F_{n, \tau}\right)$ coincide.

Consider the system of linear Diophantine equations and inequality of the form

$$
\begin{cases}M_{i}=K_{i} & (i=1, \ldots, \mu), \\ M_{i}<K_{i} & (i=\mu+1, \ldots, m)\end{cases}
$$

where for each $i=1, \ldots, m$ linear polynomials $M_{i}$ and $K_{i}$ belong to the same row of parameters of the table (6).

The row transformation of natural parameters

$$
\lambda_{s, t} \rightarrow L_{s, t} \quad(1 \leq s \leq p ; 1 \leq t \leq w)
$$

is called a parametric solution of the system (11), if the list of linear polynomials

$$
M_{i}^{\lambda_{s, t} \rightarrow L_{s, t}}, K_{i}^{\lambda_{s, t} \rightarrow L_{s, t}} \quad(i=1, \ldots, m)
$$

satisfies the system (11) for all values of parameters.

Lemma 1. For any system of linear Diophantine equations and nonequations of the form (11) it is possible to indicate the full and independent list of row transformations of natural parameters $\omega_{1}, \ldots, \omega_{\alpha}, \omega_{\alpha+1}, \ldots, \omega_{k}$ such that $\omega_{1}, \ldots, \omega_{\alpha}$ are parametric solutions of the system (11) and $\omega_{\alpha+1}, \ldots, \omega_{k}$ are not parametric solutions of the system (11) for any values of parameters.

Proof. The lemma follows from the proof of Lemma 1 of [6].

The set of words in the alphabet (2) which is obtained from the parametric word $P$ by substitution for the natural parameters of the word by all possible natural numbers will be denoted by $\langle p\rangle$.

A row transformation of natural parameters $\omega$ and an ordered list of parametric words $p_{1}, \ldots, p_{\tau}$ are called a decomposition of the parametric word $P$, if $p^{\omega}=$ $p_{1} \cdots p_{\tau}$. 
Lemma 2. For every parametric word $P$ we can indicate a finite number of decompositions $\omega_{1}, H_{1}, K_{1} ; \cdots ; \omega_{t}, H_{t}, K_{t}$ such that for any couple of words $A, B$ with condition $A B \in\langle p\rangle$ there exists the decomposition $\omega_{r}, H_{r}, K_{r}$ such that $A \in\left\langle H_{r}\right\rangle, B \in$ $\left\langle K_{r}\right\rangle$.

Proof. Let the word $P$ be of the form $P=R_{1} \cdots R_{m}$, where $R_{1}, \ldots, R_{m}$ are parametric letters of the $p$-layer alphabet. We indicate the needed decompositions. First there is the decomposition $\omega_{0}, R_{1}, \ldots, R_{s}, R_{s+1}, \ldots, R_{m}$ for the identical transformation $\omega_{0}$ and any $s$ from $0 \leq s \leq m$. Second there is the decomposition inside every letter $R_{s+1}$. Let $R_{s+1}$ be of the form $C_{q}^{\lambda_{q, t}}$. Let the letters of the $q$ th layer that occur in the word $P$ be among the letters $C_{q}^{\lambda_{q, 1}}, \ldots, C_{q}^{\lambda_{q, w}}$. Define the transformation $\omega_{1}$ as follows: $\lambda_{q, t} \rightarrow \lambda_{q, w+1}+1+\lambda_{q, w+2}$. In this case

$$
p^{\omega_{1}}=R_{1}^{\omega_{1}} \cdots R_{s}^{\omega_{1}} C_{q}^{\lambda_{q, w+1}} C_{q} C_{q}^{\lambda_{q, w+2}} R_{s+2}^{\omega_{1}} \cdots R_{m}^{\omega_{1}},
$$

where $C_{q}$ is of the form $C_{q}=T_{1} \cdots T_{h}$ where $T_{1}, \ldots, T_{h}$ are parametric letters of the $p$-1-layer alphabet. Define the decompositions of the word $C_{q}$ by the decomposition between and inside of the letters $T_{1}, \ldots, T_{h}$. Iterating the described operations we define the needed decompositions.

We prove by induction with respect to the number of the layers of the alphabet of the word $P$ that the indicated list of decompositions is as needed. If $A, B$ are words in the alphabet (2) and $A B \in\langle p\rangle$, then there exists number $s$ from $0 \leq s \leq m$ such that $A=A_{1} A_{2}, B=B_{1} B_{2}, A \in\left\langle R_{1} \cdots R_{s}\right\rangle, A_{2} B_{1} \in\left\langle R_{s+1}\right\rangle, B_{2} \in\left\langle R_{s+2} \cdots R_{m}\right\rangle$. Using the indicated supposition for words $A_{2}, B_{1}$ with condition $A_{2} B_{1} \in\left\langle R_{s+1}\right\rangle$ we find the decomposition $\omega_{r}^{\prime}, H_{r}^{\prime}, K_{r}^{\prime}$ of the parametric word $R_{s+1}$ such that $A_{2} \in$ $\left\langle H_{r}^{\prime}\right\rangle, B_{1} \in\left\langle K_{r}^{\prime}\right\rangle$, that proves the lemma.

Lemma 3. For every parametric word $P$ and any positive integer $m$ we can indicate a finite number of decompositions $\omega_{1}, p_{1,1}, \ldots, p_{1, m} ; \cdots ; \omega_{s}, p_{s, 1}, \ldots, p_{s, m}$ such that for any list of words $A_{1}, \ldots, A_{m}$ with the condition $A_{1} \cdots A_{m} \in\langle p\rangle$ there exists decomposition $\omega_{r}, p_{r, 1}, \ldots, p_{r, m}$ such that $A_{i} \in\left\langle p_{i}\right\rangle$ for all $i=1, \ldots, m$.

Proof. We prove this by induction with respect to the number of the words of the decomposition given by Lemma 2 .

The product of the finite number of row transformations of the natural parameters and replacement of the natural parameters will be called a linear transformation of the natural parameters.

Let $\mathfrak{X}$ be a free semigroup with free generators (2).

An exponential equation in free semigroup $\mathfrak{X}$ is given by the $p$-layer alphabet of normal parametric letters (7) and the equality of words

$$
\Phi\left(x_{1}, \ldots, x_{n}, \ldots \lambda_{s, t} \ldots\right)=\Psi\left(x_{1}, \ldots, x_{n}, \ldots \lambda_{s, t} \ldots\right)
$$

in this alphabet. Three types of transformation of the natural parameters of the exponential equation are defined in just the same way as the parametric equation. A linear transformation of the natural parameters $\lambda_{s, t} \rightarrow L_{s, t}$ is called a solution of the exponential equation (7), (12) whenever the words $\Phi\left(x_{1}, \ldots, x_{n}, \ldots L_{s, t} \ldots\right)$ and $\Psi\left(x_{1}, \ldots, x_{n}, \ldots L_{s, t} \ldots\right)$ coincide.

The exponential equation in a free semigroup $\mathfrak{X}$ given by the $p$-layer alphabet of normal parametric letters ( 7 ) is called a separated exponential equation, if the equality (12) is of the form

$$
\Phi\left(x_{1}, \ldots, x_{n}, C_{1}, \ldots, C_{p-1}, \ldots, \lambda_{s, t} \ldots\right)=C_{p}^{L_{p, u}}
$$


where $L_{p, u} \in \mathfrak{L}_{p}, C_{i}$ is the base of the letters of the $i$ th layer of the alphabet (7).

Statement $1, p$. The general solution of separated exponential equation in the $p$ layer alphabet is described by finite number of solutions.

Statement $2, p$. The general solution of exponential equation in the $p$-layer alphabet is described by finite number of solutions.

We prove the statements $1, p$ and $2, p$ by a joint induction with respect to the natural number $p$.

Proof of statement 1,p. First consider a separated exponential equation $\Phi\left(C_{1}, \ldots, C_{p-1}\right)=C_{p}^{\lambda_{p, m}}$ where the right hand is a parametric letter. Apply the transformation of division of the natural parameters of the form $\lambda_{q, r} \rightarrow 0, \lambda_{q, r} \rightarrow 1$, $\lambda_{q, r} \rightarrow 2+\lambda_{q, r}$ to the basic parametric letters of left hand of the equation. We obtain a list of equations of the form

$$
R_{1} R_{2} \cdots R_{d}=C_{p}^{\lambda_{m}}
$$

where every letter $R_{i}$ is either a letter from alphabet (2), or a parametric letter of the form $C_{q}^{2+\lambda_{q, r}}$ where $q<p$. Apply the transformation of division of the natural parameters $\lambda_{p, m} \rightarrow 0, \lambda_{p, m} \rightarrow 1, \ldots, \lambda_{p, m} \rightarrow 2 d, \lambda_{p, m} \rightarrow 2 d+1+\lambda_{p, m}$ to every such equation. We obtain a list of parametric equations where the first $2 d$ equations are exponential equations in the $(p-1)$-layer alphabet. Use the statement $2, p-1$ for them. The last equation has the form

$$
R_{1} R_{2} \cdots R_{d}=C_{p}^{2 d+1+\lambda_{p, m}}
$$

The exponential equation is given by the alphabet of normal parametric letters. If $R_{1}^{*}, R_{2}^{*}, \ldots, R_{d}^{*}, C_{p}^{*}$ are words in the alphabet (2) satisfying this equation, we then have $C_{p}^{*}=B A$ and for some $t$ from $1 \leq t \leq d, R_{t}^{*}=B_{2}(A B)^{l+2} A_{1}$ for certain $A=A_{1} A_{2}, B=B_{1} B_{2}$ and integer 1 . The letter $R_{t}$ has the form $C_{q}^{2+\lambda_{q, r}}$ where $q<r$. Let $\omega_{1}, H_{1}, K_{1} ; \cdots ; \omega_{s}, H_{s}, K_{s}$ are all decompositions of parametric word $C_{p}$. According to Lemma 2 we can construct them. The last statement falls into a list of equations

$$
R_{1} R_{2} \cdots R_{d}=\left(H_{i} K_{i}\right)^{2 d+1+\lambda_{p, m}} \quad(i=1, \ldots, s)
$$

where $K_{i} H_{i}=C_{q}^{\omega_{i}}$. Represent them in the form

$$
R_{1} R_{2} \cdots R_{d}=H_{i}\left(C_{q}^{\omega_{i}}\right)^{2 d+\lambda_{p, m}} K_{i} \quad(i=1, \ldots, s) .
$$

Apply the transformation of the replacement of the parameter $\lambda_{p, m}$. We obtain a list of exponential equations in the $(p-1)$-layer alphabet. Use the statement $2, p-1$.

Now let a separated exponential equation be of the general form $\Phi\left(C_{1}, \ldots, C_{p-1}\right)$ $=C_{p}^{L_{p, u}}$, where $L_{p, u} \in \mathfrak{L}_{p}$. Let $\sigma$ be the sum of the coefficients of the linear polynomial $L_{p, u}$. Using Lemma 3 we can indicate a finite number of decompositions $\omega_{1}, \ldots, \omega_{s}$ of the parametric word $\Phi\left(C_{1}, \ldots, C_{p-1}\right)$ on $\sigma$ parts such that every decomposition $\omega_{r}$ gives a system of exponential equations consisting of exponential equations in the $(p-1)$-layer alphabet and separated exponential equations of the form $\Phi\left(C_{1}, \ldots, C_{p-1}\right)=C_{p}^{\lambda_{p, m}}$. 
Proof of statement 2,p. Let an exponential equation be given by the alphabet (7) and equality (12). Let $\Phi\left(x_{1}, \ldots, x_{n}, \ldots \lambda_{s, t} \ldots\right)$ be of the form $R_{1} R_{2} \cdots R_{d}$, where $R_{i}$ are parametric letters. Let some $R_{t}$ be a letter with base $C_{p}$. Let $\omega_{1}, \ldots, \omega_{s}$ all be decompositions of the parametric word $\Psi\left(x_{1}, \ldots, x_{n}, \ldots \lambda_{s, t} \ldots\right)$ on the $d$ parametric words. According to Lemma 3 we can construct them. The alphabet (7) is an alphabet of normal parametric letters, therefore we can exclude in the list $\omega_{1}, \ldots, \omega_{s}$ of the decomposition everything interior to bases $C_{p}$ of the letters of the word $\Psi$. According to Lemma 1 every remaining decomposition gives a system of exponential equations consisting of exponential equations in the $(p-1)$-layer alphabet and separated exponential equation. Applying the statement $2, p-1$ to equations of the first case and the statement $1, p$ to equations of the second case we prove the statement $2, p$.

Let $S$ be a parametric word in the $p$-layer alphabet of normal parametric letters (7). If $\langle S\rangle$ contains only simple words and if $L=L_{1}+2$, where $L_{1} \in \mathfrak{L}_{q}$ for some $q \leq p$, then a word $S^{L}$ is called a normal power of the word $S$.

We say that the word $N$ in the $p$-layer alphabet of normal parametric letters contains the normal power $S^{L}$, if there exists a linear transformation of natural parameters $\omega$ such that $N^{\omega}=S^{L}$.

Lemma 4. Any word $N$ in the p-layer alphabet of normal parametric letters contains a finite list of normal powers $S_{1}^{L_{1}}, \ldots, S_{m}^{L_{m}}$ such that the set of words $\bigcup_{i=1}^{m}\left\langle S_{i}^{L_{i}}\right\rangle$ and the set of nonsimple words of $\langle N\rangle$ coincide.

Proof. Let $\nu$ be the number of the letters in the parametric word $N$. According to Lemma 3 there exists a finite number of decompositions, which contain all periodic words from $\langle N\rangle$ with period $i$ for all $i=1, \ldots, \nu+1$. If $i>\nu+1$, then the base of power belongs inside some parametric letter of word $N$ from the $q$ th layer. Since parametric letters of word $N$ are normal, we obtain that the exponent of power is equal to some linear polynomial from $\mathfrak{L}_{q}$.

Lemma 5. Any parametric equation $E$ can be transformed by means of a finite number of divisions, replacements and normalizations of the natural parameters into a list of parametric equations $E_{1}, \ldots, E_{s}$, given by an alphabet of the normal parametric letters. And what's more, any solution of the equation $E$ is expressed by the same solution of some equation $E_{i}$ and the transformation connecting it to E.

Proof. Suppose the parametric equation $E$ is given by an alphabet (7) and an equality $\Phi=\Psi$. Suppose $C_{1}^{\lambda_{1,1}}, \ldots, C_{m-1}^{\lambda_{m-1,1}}$ are normal parametric letters and the word $C_{m}$ is a periodic word for some value of parameters. According to Lemma 4 the word $C_{m}$ contains a finite list of normal powers, which contains all nonsimple words from $\left\langle C_{m}\right\rangle$. Applying a suitable transformation of the natural parameters to every normal power, we obtain a list of equations such that first $m$ layers of the alphabet are normal parametric letters. Continuing this process we obtain a list of equations given by an alphabet of the normal parametric letters.

A parametric equation $\Phi_{1}=\Psi_{1}$ is called a corollary of the parametric equation $\Phi=\Psi$ if there exists a linear transformation $\omega$ such that $\Omega^{\omega} \pm \Phi_{1}$ and $\Psi^{\omega} \equiv \Psi_{1}$. A list of corollaries $\Phi_{1}=\Psi_{1}, \ldots, \Phi_{\nu}=\Psi_{\nu}$ of the equation $\Phi=\Psi$ will be called full if any solution of the equation $\Phi=\Psi$ can be obtained from some $\Phi_{r}=\Psi$ by means of connected transformations. 
We say that the first variable indicates a parametric word $P$ if $p=x_{s} p_{1}$ for some parametric word $p_{1}$. We say that the first occurrence of a certain variable $x_{r}$ indicates a parametric word $P$, if $P=p_{1} x_{r} p_{2}$, where a word $p_{1}$ does not contain a variable $x_{r}$.

Lemma 6. Any parametric equation can be transformed into a full list of its corollaries which have an indicated first variable in its left side.

Proof. Let us assume that the first parametric letter in the considered word is $C_{j}^{\lambda_{j, r}}$. After the transformation of division of natural parameters $\lambda_{j, r} \rightarrow 0, \lambda_{j, r} \rightarrow 1+\lambda_{j, r}$ quantity of parametric letters in the first equality will reduce and in the second equality the number of the layer of the first letter will reduce. If we continue to apply the transformation of division the first variable will be indicated.

Lemma 7. Any parametric equation can be transformed into a full list of its corollaries which have an indicated first occurrence of a certain variable $x_{k}$ on its left side.

Proof. If the word $\Phi$ of the equation $\Phi=\Psi$ does not contain the variable $x_{k}$ we shall consider the equation $\Phi x_{k}=\Psi x_{k}$ which is equivalent to the initial one. Let us suppose that in the considered parametric word the first occurrence of a given variable $x_{k}$ is in the parametric letter $C_{j}^{\lambda_{j, k}}$ and apply the transformation of division of natural parameters $\lambda_{j, r} \rightarrow 0, \lambda_{j, r} \rightarrow \lambda_{j, r}+1+\lambda_{j, u}$, where $\lambda_{j, u}$ is a new parameter for the equation. Keeping the described division we can indicate the first occurrence of a given variable $x_{k}$.

Lemma 8. Let the sequence of parametric equations (9) be connected by the transformations $\omega_{i}$ and let the principal transformation $\Omega_{i}$ of the initial equation (2), (3) be given by the transformation (10). Then for each $i=1, \ldots, \tau$ there exists the parametric word $\Sigma_{i}$ such that

$$
\begin{aligned}
& \varphi\left(F_{1, i}, \ldots, F_{n, i}\right)=\Sigma_{i} \Phi_{i}\left(x_{1}, \ldots, x_{n}, \ldots \lambda_{s, t} \ldots\right), \\
& \psi\left(F_{1, i}, \ldots, F_{n, i}\right)=\Sigma_{i} \Psi_{i}\left(x_{1}, \ldots, x_{n}, \ldots \lambda_{s, t} \ldots\right) .
\end{aligned}
$$

Proof. We will apply induction with respect to the number of the principal transformation $\Omega_{i}$. The transformation $\omega_{i}$ belongs to either one of three types of natural parameter transformations or to one of three types of word variable transformations. It is given that $F_{k, i-1}^{\omega_{i}}=F_{k, i}, \Sigma_{i-1}^{\omega_{i}} \equiv \Sigma_{i}, \Phi_{i-1}^{\omega_{i}} \equiv \Phi_{i}, \Psi_{i-1}^{\omega_{i}} \equiv \Psi_{i}$ for each $i=1, \ldots, \tau$. Let $\omega_{i}$ be the normalization of natural parameters $\left(L_{s}+1\right) \lambda_{m, 1} \rightarrow$ $\lambda_{m, 1}$. The parametric words $\left(C_{s}\right)^{L_{s}+1}$ and $\left(\left(C_{s}\right)^{L_{s}+1}\right)^{\lambda_{m, 1}}$, which commute in $\Omega_{i-1}$, will turn into the words $\left(C_{s}\right)^{L_{s}+1}$ and $\left(C_{s}\right)^{\lambda_{m, 1}}$ in a principal transformation $\Omega_{i}$, which will also commute. For the rest of the types of transformations Lemma 8 is obvious.

Proof of Theorem 2. Clearly, the solution of the parametric equation defines concrete transformation (or a finite sequence of transformations) of this equation. As a result of the transformation we get a new parametric equation and the solution of the new equation can be obtained from the solution of the initial one by the same transformation. Let us consider the possible cases.

Let $X_{1}, \ldots, X_{n},\left\{\cdots \Lambda_{s, t} \cdots\right\}$ be a solution of the parametric equation (7), (8). According to Lemma 5 this solution determines a finite sequence of transformations of the natural parameters. They transform the equation $(7),(8)$ into a parametric 
equation which is defined in the alphabet of the normal parametric letters. The solution of the equation obtained will have the form $X_{1}, \ldots, X_{n},\left\{\cdots \Lambda_{s, t}^{\prime} \cdots\right\}$, where the natural numbers $\left\{\cdots \Lambda_{s, t} \cdots\right\}$ and $\left\{\cdots \Lambda_{s, t}^{\prime} \cdots\right\}$ are connected by the transformations from Lemma 5 .

If $(7),(8)$ is a parametric equation in the alphabet of the normal parametric letters, then according to Lemma 6 the solution $X_{1}, \ldots, X_{n},\left\{\cdots \Lambda_{s, t} \cdots\right\}$ indicates the finite sequence of the linear transformations which transfers the equation (7), (8) into a normal parametric equation.

If $(7),(8)$ is a normal parametric equation and $X_{1}=1$ is in its solution then the degenerate transformation $x_{1} \rightarrow 1$ transforms it into a parametric equation with fewer variables.

If (7), (8) is a normal parametric equation then in accordance with Lemma 6 its solutions define the finite sequence of the linear transformations transforming the equation $(7),(8)$ into an equation with indicated first occurrence of variable $x_{1}$ on the right side.

If the normal parametric equation (7), (8) is in the form of $x_{1} \Phi(\cdots)=Q x_{1} \Psi(\cdots)$ where $Q$ is a nonempty parametric word, and $\partial\left(X_{1}\right)>\partial\left(Q^{*}\right)$ where $Q^{*}$ is the result of substitution of the solution into $Q$, then the solution defines the value of parameter $\lambda$ in the parametric transformation $x_{1} \rightarrow Q^{\lambda} x_{1}$. On the other hand if $\partial\left(X_{1}\right) \leq \partial\left(Q^{*}\right)$ then according to Lemma 3 the solution defines the decomposition of the parametric word and nondegenerated transformation of the parametric equation.

The solution of the initial equation (2), (3) defines the sequence of transformations which leads us to the trivial equation for a finite number of steps. This sequence of transformations forms the principal parametric solution of the equation (2), (3).

The equality $\varphi\left(F_{1, \tau}, \ldots, F_{n, \tau}\right)=\psi\left(F_{1, \tau}, \ldots, F_{n, \tau}\right)$ follows from Lemma 8.

\section{REFERENCES}

1. R. C. Lyndon, Equations in free groups, Trans. Amer. Math. Soc. 96 (1960), 445-457. MR 27:1488

2. _ Groups with parametric exponents, Trans. Amer. Math. Soc. 96 (1960), 518-533. MR 27:1487

3. Yu. V. Matiyasevich, Enumerable sets are diophantine, (Russian), Dokl. Akad. Nauk SSSR 191 (1970), 279-282; Improved English transl., Soviet Math. Dokl. 11 (1970), 354-357. MR 41:3390

4. Yu. J. Khmelevskii, Equations in free semigroups, Trudy Mat. Inst. Steklov. 107 (1971); English transl., Proc. Steklov Inst. Math., No. 107 (1971), Amer. Math. Soc., Providence, RI, 1976. MR 51:5808

5. __ Systems of equations in a free group. I, II, Izv. Akad. Nauk SSSR Ser. Mat. 35 (1971), 1237-1268, 36 (1972), 110-179; English transl. in Math. USSR Izv. 5 (1971), 6 (1972). MR 47:1949

6. G. S. Makanin, System of standard word equations in n-layer alphabet unknowns, Sibirsk. Math. Zh. 13 (1972), 587-597; English transl. in Siberian Math. J. 13 (1972). MR 47:6861

Steklov Mathematical Institute, Vavilova 42, 117 966, Moscow GSP-1, Russia 